\title{
Hierarchical Space Shift Keying for Unequal Error Protection
}

\author{
Ronald Y. Chang, Sian-Jheng Lin, and Wei-Ho Chung, Member, IEEE
}

\begin{abstract}
In this letter, we introduce the concept of enabling unequal error protection (UEP) with space shift keying (SSK)type modulation that encodes the source information entirely in the antenna indices. Unique characteristics associated with SSKtype modulation as compared with conventional pulse/quadrature amplitude modulation (PAM/QAM) are discussed in the context of UEP design. Three general design concepts for two-level and three-level UEP are presented. Simulation results confirm the multilevel bit protection capabilities of the proposed hierarchical spatial constellation designs.
\end{abstract}

Index Terms-MIMO systems, spatial modulation, hierarchical modulation, unequal error protection.

\section{INTRODUCTION}

$\mathbf{S}$ INCE in multimedia applications some parts of the compressed video bitstream (e.g., H.264/AVC bitstream) contribute more to the perceived quality of the video than others, using unequal error protection (UEP) techniques can improve the quality of multimedia delivery over wireless fading channels. One common approach to UEP is to employ a nonuniform arrangement of the signal constellation. This approach has been studied for uncoded phase and amplitude modulation, such as phase shift keying (PSK) [1] and pulse/quadrature amplitude modulation (PAM/QAM) [2], [3], as well as for combined coding and modulation [4].

Spatial modulation (SM) [5]-[7] is a type of modulation that encodes the source information in the combination of antenna indices and traditional phase and amplitude modulations. An implementation of SM that encodes the source information fully in the antenna indices is a promising low-complexity modulation scheme for future multiple-input multiple-output (MIMO) wireless communications. This includes space shift keying (SSK) [8]-[10], generalized SSK (GSSK) [11], and Hamming code-aided SSK (HSSK) [12]. In this letter, we investigate UEP provision in combination with this class of modulation. We point out new features of spatial constellation that require new approach to UEP, and propose three easily generalizable UEP design concepts. The UEP performance of exemplary hierarchical schemes is demonstrated by simulation. The bit capacities, average power consumption, and hardware requirements (the required number of radio frequency (RF) chains at the transmitter [13]) for these schemes are also examined.

This letter is organized as follows. Sec. II presents the system description. The proposed hierarchical modulation schemes are described in Sec. III. Performance results are demonstrated in Sec. IV. Conclusion is given in Sec. V.

Manuscript received February 3, 2012. The associate editor coordinating the review of this letter and approving it for publication was J. Lee.

This research was supported by the National Science Council of Taiwan under Grant NSC 100-2221-E-001-004.

The authors are with the Research Center for Information Technology Innovation, Academia Sinica, Taipei, Taiwan (e-mail: yjrchang@gmail.com, \{sjlin,whc\}@citi.sinica.edu.tw).

Digital Object Identifier 10.1109/LCOMM.2012.070512.120242
Notations: In this letter, $\mathbf{I}_{N}$ is the $N \times N$ identity matrix, $(\cdot)^{T}$ and $(\cdot)^{H}$ the vector transpose and conjugate vector transpose, $\|\cdot\|$ the $l_{2}$-norm of a vector, $|\cdot|$ the cardinality of a set, $\mathbb{E}$ the expectation operator.

\section{SYSTEM DESCRIPTION}

We consider an uncoded MIMO system with $N_{T}$ transmit antennas and $N_{R}$ receive antennas (denoted by an $N_{T} \times N_{R}$ system). The system employs an SSK-type modulation scheme that uses solely the antenna indices to carry information. The complex baseband signal model is given by

$$
\mathbf{y}=\mathbf{H} \sqrt{E_{s}} \tilde{\mathbf{x}}+\mathbf{v}
$$

where $\mathbf{y} \in \mathbb{C}^{N_{R} \times 1}$ is the received signal, $\tilde{\mathbf{x}}$ is the $N_{T} \times 1$ transmitted symbol comprised of 1 's (corresponding to activated antennas) and 0's (corresponding to idle antennas), $\mathbf{H} \in$ $\mathbb{C}^{N_{R} \times N_{T}}$ is the flat-fading channel, $\mathbf{v} \in \mathbb{C}^{N_{R} \times 1}$ is the additive white Gaussian noise (AWGN), and $E_{s}$ is the transmit power at each transmit antenna. Channel matrix $\mathbf{H}$ has independent and identically distributed (i.i.d.) complex Gaussian entries with zero mean and covariance matrix $\sigma_{H}^{2} \mathbf{I}_{N_{R}}$, where $\sigma_{H}^{2}=1$. The channel information is assumed perfectly known to the receiver. Noise $\mathbf{v}$ has i.i.d. complex elements with zero mean and covariance matrix $\left(N_{0} / 2\right) \mathbf{I}_{N_{R}}$. Transmitted modulated symbol (constellation point) $\tilde{\mathrm{x}}$ is selected equiprobably from the modulation alphabet (constellation set) $\mathcal{A}$, where $|\mathcal{A}|=2^{m}$ for $m$ bits transmission.

Given the signal model in (1), optimal symbol detection is the maximum likelihood (ML) estimate, i.e.,

$$
\tilde{\mathbf{x}}_{\mathrm{ML}}=\arg \min _{\mathbf{x} \in \mathcal{A}}\left\|\mathbf{y}-\mathbf{H} \sqrt{E_{s}} \mathbf{x}\right\|^{2} .
$$

The system performance based on ML detection can be quantified by first deriving the pairwise error probability (PEP) and then averaging the PEP over all pairwise symbol combinations [11], [12]. The system error probability $P_{s}$ is bounded by

$$
P_{s} \leq \frac{1}{\left(\begin{array}{c}
|\mathcal{A}| \\
2
\end{array}\right)} \sum_{\substack{\mathbf{x}_{i}, \mathbf{x}_{j} \in \mathcal{A} \\
i<j}} \omega \cdot\left(\frac{E_{s}}{N_{0}}\right)^{-N_{R}} \cdot d\left(\mathbf{x}_{i}, \mathbf{x}_{j}\right)^{-N_{R}}
$$

where $\omega=\left(2 N_{T}\right)^{N_{R}} / 2$ and $d\left(\mathbf{x}_{i}, \mathbf{x}_{j}\right)$ is the Hamming distance between symbols $\mathbf{x}_{i}$ and $\mathbf{x}_{j}$. Clearly, at a given operating $E_{s} / N_{0}$ the dominant terms in (3) correspond to pairs of symbols with small pairwise Hamming distances. Hence, the minimum Hamming distance between arbitrary two symbols, $d_{\text {min }}=\min _{\mathbf{x}_{i}, \mathbf{x}_{j} \in \mathcal{A}, i<j} d\left(\mathbf{x}_{i}, \mathbf{x}_{j}\right)$, determines the system performance.

\section{Hierarchical Space Shift Keying}

\section{A. Principles of Hierarchical Modulation}

The technique of UEP in combination with phase and amplitude modulation such as PAM and QAM employs 
a nonuniform constellation placement so that constellation points labelled by distinct values of the higher priority bit(s) are separated by greater Euclidean distances. To achieve UEP with hierarchical SSK-type modulation, intuitively, we may consider a similar strategy of nonuniform constellation placement yet in terms of the Hamming distance. However, new features of spatial constellation call for new design solutions, as discussed below.

1) While the signal constellation of PAM/QAM is one/twodimensional, the spatial constellation of SSK-type modulation is $N_{T}$-dimensional. More specifically, the Euclidean distance is defined on $\mathbb{R}^{1}$ (PAM) or $\mathbb{R}^{2}(\mathrm{QAM})$, whereas the Hamming distance is defined on $\{0,1\}^{N_{T}}$. As a result of multidimensionality, it is not always possible to group spatial constellation points into subsets so that inter-subset distances are greater than intrasubset distances. For example, if we try to group symbols $[1,1,0,0,0]^{T},[1,0,0,1,0]^{T},[0,1,1,0,0]^{T}$, and $[0,0,1,1,0]^{T}$ into two subsets of size 2 , we will end up with intra-subset distances being 2 and inter-subset distances being 2 and 4 (e.g., by placing the former two into a subset and the latter two into the other). The inter-subset distance is not greater than the intra-subset distance for some symbol combinations.

2) Since the Euclidean distance is defined on the set of real numbers, it can be adjusted quite arbitrarily for PAM/QAM. On the contrary, the adjustment of the Hamming distance defined on binary numbers is subject to the constraint of available number of transmit antennas and the set of all possible antenna indices for SSK-type modulation. Furthermore, the Hamming distances can only be positive integers and the ratios between distances have limited possibilities.

An example of conventional hierarchical modulation is shown in Fig. 1(a) for 16-HQAM (hierarchical QAM). The distance factor $\alpha=a / b$ is adjusted to yield different degrees of two-level protection. When $\alpha>1$, UEP is provided for two high-priority (HP) bits, which map to one of the four quadrants, and two low-priority (LP) bits, which map to a signal point in a quadrant. Given the same average signal power, increasing $\alpha$ increases the HP protection but decreases the LP protection. Note that three-level protection with 64HQAM can be quantified similarly, where two distance factors are defined and three priorities, i.e., HP, mid-priority (MP), and LP, are supported [2].

Due to the multidimensionality of spatial constellations, the distance factors for hierarchical SSK-type modulation cannot be defined in as visually intuitive way as for HQAM. Motivated by the fact that $d_{\min }$ determines the error-rate performance as presented in Sec. II, we propose to define the distance factors as

$$
\begin{aligned}
& \alpha=a / b=d_{\min }^{(\mathrm{HP})} / d_{\min }^{(\mathrm{LP})} \\
& \alpha=a / b=d_{\min }^{(\mathrm{HP})} / d_{\min }^{(\mathrm{MP})}, \quad \beta=b / c=d_{\min }^{(\mathrm{MP})} / d_{\min }^{(\mathrm{LP})}
\end{aligned}
$$

for two-level and three-level protection, respectively, where $d_{\text {min }}^{(*)}$ represents $d_{\min }$ between constellation points labelled by distinct values of the bit(s) of priority level $*$. This consideration circumvents the issue of multidimensionality of spatial

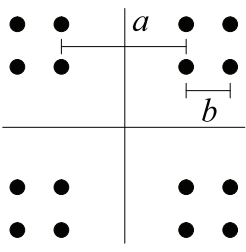

(a)

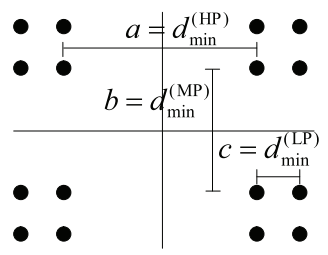

(b)
Fig. 1. Hierarchical constellations. (a) 16-HQAM for two-level protection. (b) $(4,1)(3,1)(3,2)$ H-HSSK for three-level protection (notations to be explained in Sec. III-B).

constellation and facilitates the UEP design for hierarchical SSK-type modulation. An example is shown in Fig. 1(b), which will be further examined as we present exemplary UEP designs in the following.

\section{B. UEP Designs}

We consider enabling UEP with GSSK [11] (including SSK) and HSSK [12]. GSSK employs a fixed number of $n_{t}$ 1 's and $N_{T}-n_{t}$ 0's in each modulation symbol (SSK is a special case with $n_{t}=1$ ). For example, to support 2 bits per transmission in a system with $N_{T}=4$, the SSK alphabet is

$$
\mathcal{A}^{(\mathrm{SSK})}=\left\{[0,0,0,1]^{T},[0,0,1,0]^{T},[0,1,0,0]^{T},[1,0,0,0]^{T}\right\} .
$$

HSSK employs a varied number of 1's for each modulation symbol based on the Hamming code (in general, binary linear block code) construction technique. For example, to support 3 bits per transmission in the same system with $N_{T}=4$, the HSSK alphabet incorporates the eight codewords of the $(4,3)$ binary parity check code ${ }^{1}$ with the last bit of each codeword complemented, and is given by

$$
\begin{aligned}
\mathcal{A}^{(\text {HSSK })=}\{ & {[0,0,0,1]^{T},[0,0,1,0]^{T},[0,1,0,0]^{T},[1,0,0,0]^{T}, } \\
& {\left.[0,1,1,1]^{T},[1,0,1,1]^{T},[1,1,0,1]^{T},[1,1,1,0]^{T}\right\} . }
\end{aligned}
$$

To exemplify our UEP designs with GSSK and HSSK, we consider offering two-level protection for a source symbol consisting of $1 \mathrm{HP}$ bit and $2 \mathrm{LP}$ bits in a system with $N_{T}=7$. Three design concepts are introduced, the first two based on hierarchical HSSK (H-HSSK) and the third one based on hierarchical GSSK (H-GSSK). ${ }^{2}$

Design Concept 1 (H-HSSK, Concatenation): The first UEP design with HSSK is to map the HP/LP bit(s) to an alphabet constructed from a code with a larger/smaller $d$ and concatenate the two alphabets to form the H-HSSK alphabet. The idea is similar to the concatenation of codes (see, e.g., [14, Sec. 6.2.1]) although notable distinctions can be made between UEP with uncoded modulation (our consideration) and with channel coding in terms of channel model, detection, and error patterns. An example of this UEP design is shown in Fig. 2(a), where the HP bit maps to the two codewords of the $(4,1)$ code $[0,0,1,1]^{T},[1,1,0,0]^{T}$ with $d=4$, and

\footnotetext{
${ }^{1}$ We use the notation $(n, k)$ for binary linear block codes with block length $n$, message length $k$, and an implicit minimum distance $d$. For example, $(n, n-1)$ is binary parity check code with $d=2$, and $(n, 1)$ is repetition code with $d=n$.

${ }^{2}$ The term H-GSSK is used since it is developed based on GSSK. However, unlike GSSK, symbols of H-GSSK may contain a varied number of 1's.
} 


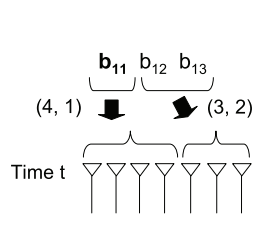

(a)

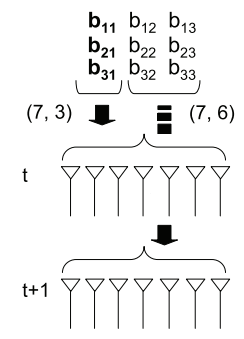

(b)

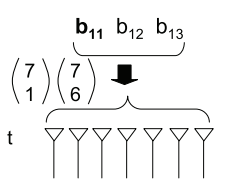

(c)
TABLE I

Hierarchical SSK-TyPe Modulation Alphabet For 3 Bits ( 1 HP (IN BoldFACE), 2 LP) Transmission IN A System With $N_{T}=7$

\begin{tabular}{|c|c|c|}
\hline Source bits & $(4,1)(3,2)$ H-HSSK symbols & $\left(\begin{array}{l}7 \\
1\end{array}\right)\left(\begin{array}{l}7 \\
6\end{array}\right)$ H-GSSK symbols \\
\hline \hline $\mathbf{0 0 0}$ & {$[0,0,1,1,0,0,0]^{T}$} & {$[1,0,0,0,0,0,0]^{T}$} \\
\hline $\mathbf{0 0 1}$ & {$[0,0,1,1,0,1,1]^{T}$} & {$[0,1,0,0,0,0,0]^{T}$} \\
\hline $\mathbf{0 1 0}$ & {$[0,0,1,1,1,0,1]^{T}$} & {$[0,0,1,0,0,0,0]^{T}$} \\
\hline $\mathbf{0 1 1}$ & {$[0,0,1,1,1,1,0]^{T}$} & {$[0,0,0,1,0,0,0]^{T}$} \\
\hline $\mathbf{1 0 0}$ & {$[1,1,0,0,0,0,0]^{T}$} & {$[0,1,1,1,1,1,1]^{T}$} \\
\hline $\mathbf{1 0 1}$ & {$[1,1,0,0,0,1,1]^{T}$} & {$[1,0,1,1,1,1,1]^{T}$} \\
\hline $\mathbf{1 1 0}$ & {$[1,1,0,0,1,0,1]^{T}$} & {$[1,1,0,1,1,1,1]^{T}$} \\
\hline $\mathbf{1 1 1}$ & {$[1,1,0,0,1,1,0]^{T}$} & {$[1,1,1,0,1,1,1]^{T}$} \\
\hline
\end{tabular}

Fig. 2. UEP designs with (a)(b) H-HSSK and (c) H-GSSK for 3 bits (1 HP (in boldface), 2 LP) per source symbol transmission in a system with $N_{T}=7$.

the two LP bits map to the four codewords of the $(3,2)$ code $[0,0,0]^{T},[0,1,1]^{T},[1,0,1]^{T},[1,1,0]^{T}$ with $d=2$. The concatenated codewords form the eight symbols of the UEP scheme, denoted by $(4,1)(3,2)$ H-HSSK, as shown in Table I. Binary-coded bit mapping is adopted for the binary symbols. For this scheme, $d_{\min }^{(\mathrm{HP})}=4, d_{\min }^{(\mathrm{LP})}=2$, and $\alpha=4 / 2=2$. Clearly, this construction principle can be customized to support different HP and/or LP bit rates.

This construction principle can also be generalized to provide three-level protection. For instance, $(4,1)(3,1)(3,2) \mathrm{H}$ HSSK can support $1 \mathrm{HP}, 1 \mathrm{MP}$, and $2 \mathrm{LP}$ bits transmission in a system with $N_{T}=10$. This specific scheme has eight constellation points as illustrated in Fig. 1(b) where we show a 2-D representation of the multidimensional constellation. The HP bit maps to the left and right half planes, the MP bit maps to the upper and lower half planes, and the two LP bits map to signal points in a quadrant. For this scheme, $\alpha=4 / 3=1.33$ and $\beta=3 / 2=1.5$.

Design Concept 2 (H-HSSK, Time-sharing): The second UEP design with HSSK is to collect several source symbols and transmit bits of different priorities separately. As shown in Fig. 2(b), the HP (LP) bits map to the nonhierarchical HSSK alphabet constructed by the $(7,3)((7,6))$ code with $d=4$ $(d=2)$ and transmitted at time $t(t+1)$ ("time sharing"). This scheme is denoted by $(7,3)+(7,6) \mathrm{H}$-HSSK, with $d_{\min }^{(\mathrm{HP})}=4$, $d_{\min }^{(\mathrm{LP})}=2$, and $\alpha=4 / 2=2$. Clearly, this design strategy can easily be generalized to provide three-level protection and support different bit rates.

Design Concept 3 (H-GSSK): The UEP design based on GSSK is to map the HP bit to two groups, one consisting of GSSK symbols with $n_{t} 1$ 's and the other consisting of "complemented" GSSK symbols with $N_{T}-n_{t} 1$ 's, and map the LP bits to different symbols in each group. The value of $n_{t}$ is chosen such that $\left(\begin{array}{c}N_{T} \\ n_{t}-1\end{array}\right)<2^{m} \leq\left(\begin{array}{c}N_{T} \\ n_{t}\end{array}\right)$ and $n_{t} \leq$ $N_{T} / 2-1$ if there are $m$ LP bits, where the latter ensures $\alpha \geq 1$ to achieve UEP. For the example in Fig. 2(c), we have $n_{t}=1$, and the eight symbols of this specific scheme, denoted by $\left(\begin{array}{l}7 \\ 1\end{array}\right)\left(\begin{array}{l}7 \\ 6\end{array}\right)$ H-GSSK, are shown in Table I. As can be seen, $d_{\mathrm{min}}^{(\mathrm{HP})}=N_{T}-2 n_{t}=5, d_{\mathrm{min}}^{(\mathrm{LP})}=2$, and $\alpha=5 / 2=2.5$ for this scheme. This design strategy applies to two-level protection only and supports HP bit rate of one.

\section{Simulation Results}

Based on the three design concepts, different hierarchical schemes can be produced with various degrees of protection, capacities for each bit priority, system requirements, and performance. To reflect typical application scenarios and achieve meaningful comparisons with nonhierarchical schemes, we produce different hierarchical schemes with the following considerations: 1) $d_{\min }^{(\mathrm{HP})} \geq d_{\mathrm{min}}^{(\mathrm{MP})} \geq d_{\min }^{(\mathrm{LP})}=2$, and 2) $C^{(\mathrm{HP})} \leq C^{(\mathrm{MP})} \leq C^{(\mathrm{LP})}$, where $C$ stands for the capacity for the corresponding bit priority. The considered hierarchical schemes are summarized in Table II, and the bit-error-rate (BER) performance versus signal-to-noise ratio (SNR) results are shown in Figs. 3-5 (BER values at $\mathrm{SNR}=16 \mathrm{~dB}$ are included in Table II to facilitate cross-comparison), where the SNR is defined as $E_{s} \cdot \mathbb{E}\left[\tilde{\mathbf{x}}^{H} \tilde{\mathbf{x}}\right] / N_{0}$. ML detection is adopted for all schemes in comparison.

In Figs. 3 and 4, we plot the BER performance for two-level $\mathrm{H}-\mathrm{HSSK}$ and H-GSSK in comparison with nonhierarchical GSSK/HSSK schemes as well as conventional HQAM, with fixed target bit rates (5 and 2, respectively). As seen in Fig. 3, H-GSSK9 and H-HSSK1 show comparable performance, both outperforming 32-HQAM (with gray-coded bit mapping) at the same aggregate rate (1 HP and 4 LP bits). Comparing H-HSSK1 and H-HSSK4 in Figs. 3-4, and also H-HSSK1-4 in Table II, we see that with the same $d_{\min }^{(\mathrm{LP})}=2$, increasing $d_{\min }^{(\mathrm{HP})}$ (or $\alpha$ ) improves the HP protection significantly and the LP protection slightly at the tradeoff of reduced LP capacity. The slight improvement of the LP protection despite the same $d_{\mathrm{min}}^{(\mathrm{LP})}=2$ is because a larger $d_{\mathrm{min}}^{(\mathrm{HP})}$ also increases the average distance between symbols labelled by distinct values of the LP bits. The HP/LP performance of H-HSSK7-8 schemes is demonstrated by the nonhierarchical HSSK schemes in Fig. 4. Comparing H-HSSK7 and H-HSSK2 in Table II with the same $d_{\mathrm{min}}^{(\mathrm{LP})}$ and $d_{\mathrm{min}}^{(\mathrm{HP})}$, H-HSSK7 achieves higher capacity at the cost of minor BER performance degradation and a larger number of required RF chains. The analytical BER performance upper bound, derived by summing over pairs of symbols labelled by distinct values of the HP/LP bits in (3), is also plotted in Fig. 4 for H-HSSK4, which is loose for low SNR and tighter for high SNR. ${ }^{3}$

Fig. 5 shows the BER performance for three-level H-HSSK5 at a target bit rate of 4 . The result confirms the multilevel bit protection capabilities of the proposed hierarchical design. Comparing H-HSSK5 and H-HSSK6 in Table II, we see that H-HSSK6 achieves notably improved HP protection with a larger $\alpha$, and slightly better MP and LP protection with the same $\beta$, at the cost of lower total capacity.

\footnotetext{
${ }^{3} \mathrm{~A}$ suggestion on tightening the bound is given in [12].
} 
TABLE II

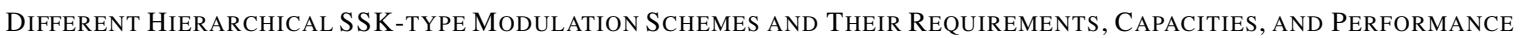

\begin{tabular}{|c|c|c|c|c|c|c|c|c|c|c|c|c|}
\hline \multirow{2}{*}{$\begin{array}{l}\text { Scheme (1-8 H-HSSK, } \\
9-10 \text { H-GSSK) }\end{array}$} & \multirow[t]{2}{*}{$N_{T}$} & \multirow{2}{*}{$\begin{array}{l}\text { Req. \# RF } \\
\text { chains }\end{array}$} & \multirow{2}{*}{$\begin{array}{l}\text { Avg. symbol } \\
\text { power }\end{array}$} & \multirow[t]{2}{*}{$\alpha$} & \multirow[t]{2}{*}{$\beta$} & \multicolumn{4}{|c|}{ Capacity (avg. \# bits/symbol) } & \multicolumn{3}{|c|}{ BER performance $(\mathrm{SNR}=16 \mathrm{~dB})$} \\
\hline & & & & & & $\mathrm{HP}$ & MP & LP & Total & HP & MP & LP \\
\hline 1. $(2,1)(5,4)$ & $\overline{7}$ & $\overline{5}$ & 3.5 & $\overline{1}$ & - & $\overline{1}$ & 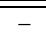 & 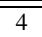 & $\overline{5}$ & $1.9 \times 10^{-4}$ & - & $8.1 \times 10^{-4}$ \\
\hline 2. $(3,1)(4,3)$ & 7 & 5 & 3.5 & 1.5 & - & 1 & - & 3 & 4 & $3.0 \times 10^{-5}$ & - & $4.5 \times 10^{-4}$ \\
\hline 3. $(4,1)(3,2)$ & 7 & 4 & 3.5 & 2 & - & 1 & - & 2 & 3 & $5.8 \times 10^{-6}$ & - & $3.0 \times 10^{-4}$ \\
\hline 4. $(5,1)(2,1)$ & 7 & 4 & 3.5 & 2.5 & - & 1 & - & 1 & 2 & $1.5 \times 10^{-6}$ & - & $1.5 \times 10^{-4}$ \\
\hline 5. $(4,1)(3,1)(3,2)$ & 10 & 6 & 5 & 1.33 & 1.5 & 1 & 1 & 2 & 4 & $9.3 \times 10^{-6}$ & $4.8 \times 10^{-5}$ & $7.1 \times 10^{-4}$ \\
\hline 6. $(5,1)(3,1)(2,1)$ & 10 & 6 & 5 & 1.67 & 1.5 & 1 & 1 & 1 & 3 & $2.1 \times 10^{-6}$ & $4.6 \times 10^{-5}$ & $3.6 \times 10^{-4}$ \\
\hline 7. $(7,4)+(7,6)$ & 7 & 7 & 3.5 & 1.5 & - & 1.33 & - & 4 & 5.33 & $8.8 \times 10^{-5}$ & - & $1.4 \times 10^{-3}$ \\
\hline 8. $(7,3)+(7,6)$ & 7 & 7 & 3.5 & 2 & - & 1.5 & - & 3 & 4.5 & $1.9 \times 10^{-5}$ & - & $1.4 \times 10^{-3}$ \\
\hline 9. $\left(\begin{array}{l}7 \\
2\end{array}\right)\left(\begin{array}{l}7 \\
5\end{array}\right)$ & 7 & 5 & 3.5 & 1.5 & - & 1 & - & 4 & 5 & $1.6 \times 10^{-4}$ & - & $7.2 \times 10^{-4}$ \\
\hline 10. $\left(\begin{array}{l}7 \\
1\end{array}\right)\left(\begin{array}{l}7 \\
6\end{array}\right)$ & 7 & 6 & 3.5 & 2.5 & - & 1 & - & 2 & 3 & $4.1 \times 10^{-6}$ & - & $3.0 \times 10^{-4}$ \\
\hline
\end{tabular}

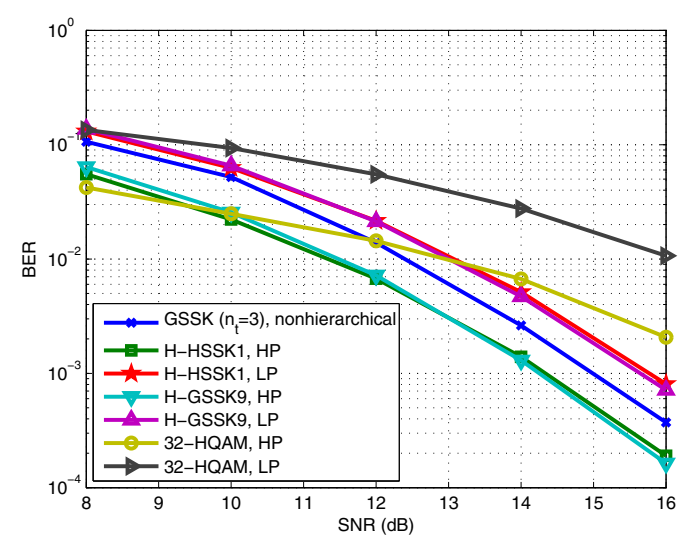

Fig. 3. BER performance for two-level hierarchical SSK-type modulation schemes $(7 \times 7$ system $)$ versus nonhierarchical scheme $(7 \times 7$ system $)$ and 32-HQAM $(1 \times 7$ system $)$, for 5 bits/s/Hz transmission.

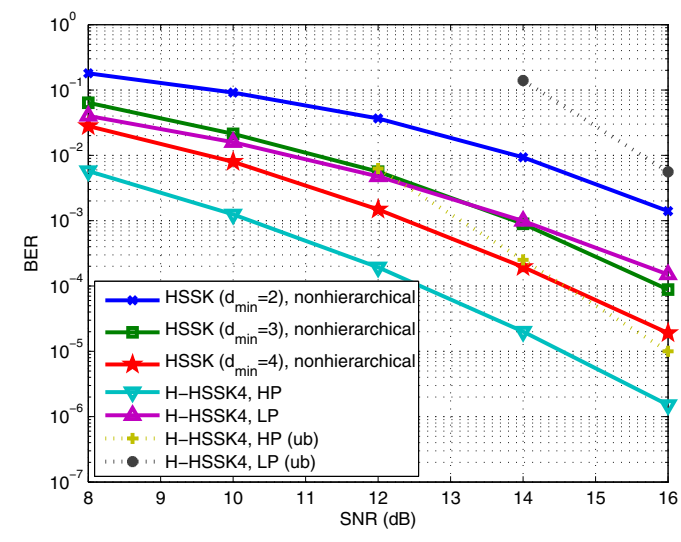

Fig. 4. BER performance for two-level hierarchical SSK-type modulation scheme $(7 \times 7$ system) versus nonhierarchical scheme $(7 \times 7$ system $)$, for 2 bits/s/Hz transmission.

\section{CONCLUSION}

Hierarchical SSK-type modulation schemes have been proposed. The nonuniform arrangement of multidimensional spatial constellation was developed and visualized through an illustrative example. A detailed study of the performance, requirements (hardware and power), and capacities of various hierarchical schemes offered insights into the use of spatial modulation for multimedia delivery over low-bit-rate wireless channels.

\section{REFERENCES}

[1] M. B. Pursley and J. M. Shea, "Adaptive nonuniform phase-shift-key modulation for multimedia traffic in wireless networks," IEEE J. Sel. Areas Commun., vol. 18, no. 8, pp. 1394-1407, Aug. 2000.

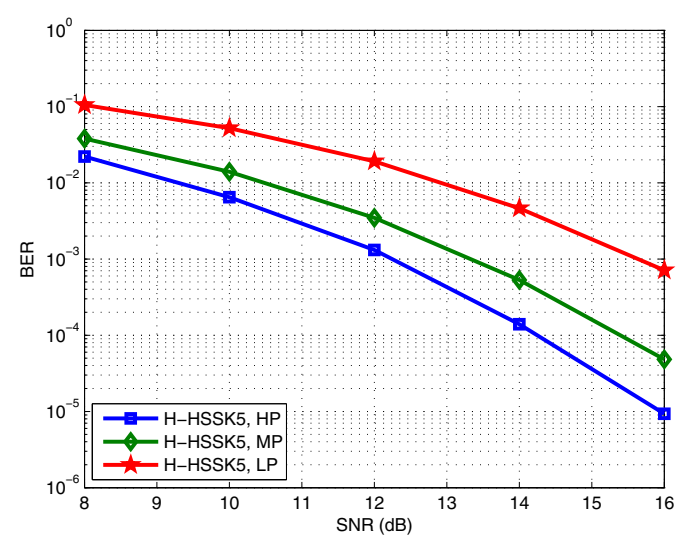

Fig. 5. BER performance for three-level hierarchical SSK-type modulation scheme $(10 \times 10$ system $)$, for $4 \mathrm{bits} / \mathrm{s} / \mathrm{Hz}$ transmission.

[2] B. Barmada, M. M. Ghandi, E. V. Jones, and M. Ghanbari, "Prioritized transmission of data partitioned H.264 video with hierarchical QAM," IEEE Signal Process. Lett., vol. 12, no. 8, pp. 577-580, Aug. 2005.

[3] P. K. Vitthaladevuni and M.-S. Alouini, "A recursive algorithm for the exact BER computation of generalized hierarchical QAM constellations," IEEE Trans. Inf. Theory, vol. 49, no. 1, pp. 297-307, Jan. 2003.

[4] A. R. Calderbank and N. Seshadri, "Multilevel codes for unequal error protection," IEEE Trans. Inf. Theory, vol. 39, no. 4, pp. 1234-1248, July 1993.

[5] R. Y. Mesleh, H. Haas, S. Sinanović, C. W. Ahn, and S. Yun, "Spatial modulation," IEEE Trans. Veh. Technol., vol. 57, no. 4, pp. 2228-2241, July 2008.

[6] E. Basar, U. Aygolu, E. Panayirci, and H. V. Poor, "Space-time block coded spatial modulation," IEEE Trans. Commun., vol. 59, no. 3, pp. 823-832, Mar. 2011.

[7] S. Sugiura, S. Chen, and L. Hanzo, "Generalized space-time shift keying designed for flexible diversity-, multiplexing- and complexity-tradeoffs," IEEE Trans. Wireless Commun., vol. 10, no. 4, pp. 1144-1153, Apr. 2011.

[8] J. Jeganathan, A. Ghrayeb, L. Szczecinski, and A. Ceron, "Space shift keying modulation for MIMO channels," IEEE Trans. Wireless Commun., vol. 8, no. 7, pp. 3692-3703, July 2009.

[9] M. Di Renzo and H. Haas, "A general framework for performance analysis of space shift keying (SSK) modulation for MISO correlated Nakagami-m fading channels," IEEE Trans. Commun., vol. 58, no. 9 , pp. 2590-2603, Sep. 2010.

[10] -, "Space shift keying (SSK-) MIMO over correlated Rician fading channels: Performance analysis and a new method for transmitdiversity," IEEE Trans. Commun., vol. 59, no. 1, pp. 116-129, Sep. 2011.

[11] J. Jeganathan, A. Ghrayeb, and L. Szczecinski, "Generalized space shift keying modulation for MIMO channels," in Proc. 2008 IEEE PIMRC, pp. $1-5$.

[12] R. Y. Chang, S.-J. Lin, and W.-H. Chung, "New space shift keying modulation with Hamming code-aided constellation design," IEEE Wireless Commun. Lett., vol. 1, no. 1, pp. 2-5, Feb. 2012.

[13] A. F. Molisch and M. Z. Win, "MIMO systems with antenna selection," IEEE Microwave Mag., vol. 5, no. 1, pp. 46-56, Mar. 2004.

[14] R. H. Morelos-Zaragoza, The Art of Error Correcting Coding, 2nd edition. Wiley, 2006. 